\section{NEW GENERATION OF BONDING AGENTS}

Shofu's BeautyOrtho Bond, known as BOB, is the first of a new generation of bonding agents developed specifically for bonding orthodontic brackets.

When teeth are etched, the material penetrates the undercut areas of the tooth, often resulting in difficulty removing the brackets and harm to the tooth structure. With BOB, pre-etching is not necessary as the primer is made up of 6-methacryloxyhexylphosphonoacetate which chemically adheres to the enamel. In addition, the S-PEG filler component of BOB releases fluoride on a continuous basis, meaning that the material is constantly re-charged providing ultimate protection for the teeth.

BOB provides good handling properties and easy debonding at the end of treatment without damaging the tooth structure. Simple to use, you apply the primer, air dry and extrude the paste. Once the bracket is in position, cure to secure. As BOB paste is light cured you have plenty of time to position the bracket accurately before curing.

Reader response number 61

\section{COMFORTABLE STEREOSCOPIC VISION}

Carl Zeiss' OPMI Pico magnification device has been developed exclusively for dentistry, and the optical quality enables comfortable stereoscopic vision, depth orientation and high contrast.

The integrated Superlux 180 xenon light source delivers daylight quality illumination and the MORA interface dramatically improves the posture of the dental professional, eradicating those back problems that commonly plague the profession.

By employing the OPMI Pico, dentists ultimately increase their diagnostic and treatment skills, seeing and identifying problems with a level of clarity not previously possible, which in turn improves the patient journey and cuts down the timescale needed for each patient.

Reader response number 62

\section{MINIMAL INTERVENTION TREATMENT TECHNIQUES}

Ultrasonic scalers are in daily use in the dental surgery, providing a patientfriendly and efficient way to meet the challenges of perio, endo and minimal intervention treatment techniques.

The Varios 970 ultrasonic scaler, from NSK, can be used for these treatments simply by exchanging the tip and with a choice of over 70 tips, there is always one for the procedure you are undertaking.

Each Varios 970 LUX is supplied, as standard, with a twin LED handpiece, three tips, three tip wrenches and an autoclavable storage container for handpiece, tips and wrenches.

The twin LEDs deliver shadow-free illumination to the entire treatment field, allowing faster and more accurate treatment. The Varios 970 has a very useful second solution container, allowing you to easily change irrigants during a treatment with a dial control. The unit also has three power ranges, Perio, Endo and General, that are eas-

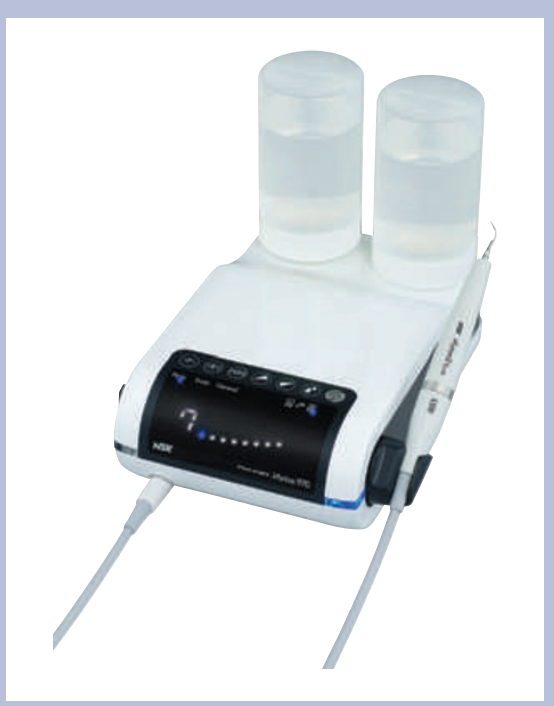

ily selected with the push of a button.

The Varios 970 incorporates the brand new NSK iPiezo control board with the very latest and most advanced standards in auto-feedback technology, making the performance smoother and more efficient.

Reader response number 63

\title{
STRENGTH, LIGHTNESS AND MANOEUVRABILITY
}

Goldspeed is a new range of handpieces by Castellini. Manufactured according to the highest standards of micromechanics, they have titanium heads and handgrips for strength and lightness, contra-angled for balance and manoeuvrability, and high-intensity fibre optic lighting for long-lasting, white illumination.

\section{SOFT-GRIP SILICONE HANDLES}

Dentsply's Flexichange range is ergonomically designed with soft-grip silicone handles, to fit perfectly in your hand. With a wide grip at the working end and a narrow centre, the dimpled design helps stop hand fatigue and prevent 'pinching' of the handle, thus improving grip and rotational control.

The range includes a selection of scalers, curettes and hoes, including a number of instruments also available with slimmer tips allowing for finer
Viable for general, restorative, endodontic, prosthetic and surgical fields, they are easily compatible with all ISO 3964 micromotor couplings. Sanitation is also integral to the handpieces as they are fully autoclaveable and include a device to prevent the retraction of contaminants.

Reader response number 64 